Revue d'histoire de l'Amérique française

\#IS REVUE D.HISTOIRE DE L'AMÉRIQUE FRANÇAISE

\title{
Du système colonial des peuples modernes - Un inédit de Rameau de Saint-Père
}

\section{Pierre Trépanier}

Volume 36, numéro 1, juin 1982

URI : https://id.erudit.org/iderudit/304031ar

DOI : https://doi.org/10.7202/304031ar

Aller au sommaire du numéro

Éditeur(s)

Institut d'histoire de l'Amérique française

ISSN

0035-2357 (imprimé)

1492-1383 (numérique)

Découvrir la revue

Citer cet article

Trépanier, P. (1982). Du système colonial des peuples modernes — Un inédit de Rameau de Saint-Père. Revue d'histoire de l'Amérique française, 36(1), 55-74. https://doi.org/10.7202/304031ar d'utilisation que vous pouvez consulter en ligne.

https://apropos.erudit.org/fr/usagers/politique-dutilisation/ 


\title{
DU SYSTÈME COLONIAL DES PEUPLES MODERNES UN INÉDIT DE RAMEAU DE SAINT-PËRE
}

\author{
PIERRE TRÉPANIER* \\ Département d'histoire \\ Université de Montréal
}

Les hasards de la recherche réservent parfois d'agréables surprises. Un griffonnage laconique se trouve à l'origine de notre trouvaille. Parmi les brouillons de Rameau de Saint-Père à l'Université de Moncton, a retenu notre attention une table des matières avec, dans le coin gauche supérieur, la mention: «Travail déposé à l'Institut pour le concours Léon Faucher...»' 'Les archives de l'Institut de France conservent en effet un travail de Rameau. Il s'agit d'un fort manuscrit in-quarto de 707 pages portant le numéro quatre $^{2}$. La question mise au concours sert de titre, avec en épigraphe cette phrase de Vauban: "Qui peut entreprendre quelque chose de plus grand et de plus utile qu'une colonie?» Aucune étude, aucune bibliographie ne mentionne cet inédit.

Le prix Léon-Faucher est décerné tous les trois ans par l'Académie des Sciences morales et politiques à «l'auteur du meilleur mémoire sur une question d'économie politique ou sur la vie d'un économiste célèbre, soit français, soit étranger» ${ }^{3}$. Le 9 juin 1866 , l'Académie décida de faire porter le concours sur le sujet du

* Avec le concours de Lise Trépanier. Nous remercions le Conseil de recherches en sciences humaines du Canada et l'Université de Montréal (programme CAFIR) pour leur aide financière. Toute notre gratitude aussi à Mme Lafitte-Larnaudie, conservateur des archives de l'Institut de France, dont la bienveillante collaboration nous a été précieuse. Père, 2.4-8.

Université de Moncton, Centre d'études acadiennes, fonds Rameau de Saint-

2 En fait, 732 pages compte tenu des erreurs de pagination. Les documents dont la liste suit perdent beaucoup d'intérêt du fait qu'ils ne sont que des ébauches et brouillons du manuscrit conservé à l'Institut. Paris, Archives de la famille Decencière Ferrandière, dossier "Travail sur les colonies anglaises», cahiers L à V; dossier "Travail sur les colonies espagnoles et hollandaises». Au Centre d'études acadiennes, de nombreuses pièces sous les cotes 2.2-13, 2.4-8 et 2.10-23. Montréal, Archives Pierre et Lise Trépanier, quelques notes éparses, don de M. F. Millou, petit-fils de Rameau. Quant au manuscrit, il est conservé à l'Institut de France, sous la cote 1G3 des Archives de l'Académie des Sciences morales et politiques.

3 Annuaire de l'Institut, 1975, 285. A.-C. Franquet de Franqueville, Le premier siècle de l'Institut de France (Paris, 1896), II: 446. Institut de France, Académie des Sciences morales et politiques, Concours de l'Académie, 1834-1900 (Paris, 1901), 54, 75. Archives de l'Académie des Sciences morales et politiques, «Procès-verbal», séance du 19 mars 1870. - Léon Faucher (1804-1854), journaliste et économiste, a été ministre en 1848.

RHAF, vol. 36, no 1 , juin 1982 
système colonial des peuples modernes. Quatre concurrents étaient en lice. Paul Leroy-Beaulieu, le seul à avoir quelque célébrité, remporta le prix ${ }^{4}$. Rameau avait déposé son manuscrit le 31 décembre 1868 , terme du concours. Faute de temps, il n'avait pu donner aux appendices le développement qu'il aurait souhaité. Selon le rapport lu à la séance du 19 mars 1870 par le rapporteur, Émile Levasseur, les mémoires présentés ont impressionné le jury, à l'exception de celui qui porte le numéro un, au triple point de vue de l'étendue, de la variété et du mérite ${ }^{5}$. La palme est allée au mémoire de Leroy-Beaulieu parce qu'il est le seul qui, «sans être complet, ait cependant embrassé le sujet dans son ensemble, et traité presque toutes les parties importantes d'une manière satisfaisante» ${ }^{6}$.

On notera que Levasseur et Leroy-Beaulieu joueront un rôle important au sein de la Société d'économie sociale, fondée par Le Play et dont Rameau était aussi membre. Cette société, bien que là ne fut pas son but premier, partageait, avec de nombreuses autres compagnies savantes, la fonction de centre de diffusion des idées d'expansion coloniale ${ }^{7}$. Avec les années, grâce aux efforts de

4 Né en 1843, mort en 1916, Leroy-Beaulieu, économiste libre-échangiste, est passé à l'histoire comme l'un des principaux représentants de l'école libérale. On lui doit la fondation du second Économiste français, le premier ayant été lancé par Jules Duval, ami de Rameau et fervent partisan de la colonisation de l'Algérie. Le mémoire de Leroy-Beaulieu a été publié sous le titre De la colonisation chez les peuples modernes (Paris, Guillaumin, 1874). L'ouvrage a connu un certain succès puisqu'il a été réédité en 1882, 1886 et 1891 . Leroy-Beaulieu a été élu à l'Académie des Sciences morales et politiques (1878).

5 E. Levasseur, «Rapport sur le concours pour le prix fondé par Mme Léon Faucher», Mémoires d'e l'Académie des Sciences morales et politiques, 13 (1870): 472.

6 E. Levasseur, «Rapport...», 510.

7 Le Play lui-même a assez peu écrit sur la colonisation, mais ses idées ont fait école. Rameau les reprend dans ses écrits, qu'il s'agisse d'une convergence intellectuelle ou d'un emprunt. Dans l'Organisation du Travail (1870), Le Play soutient qu'une «race établie sur un sol entièrement approprié à la culture ne reste prospère qu'en gardant la fécondité et en organisant, par suite, un régime régulier d'émigration». Dans la Réforme sociale en France (8 éditions de 1864 à 1901), il rejette l'opinion suivant laquelle «l'impuissance coloniale de notre race provient d'une infériorité organique, contre laquelle on tenterait vainement de réagir; à ce point de vue et malgré ses autres aptitudes, la nation française serait absolument impropre à la colonisation». La faculté d'expansion coloniale repose sur les «efforts combinés des familles-souches et des pouvoirs publics de la métropole. Toute race féconde continue à fonder de nos jours des colonies prospères, malgré certaines défaillances des gouvernants et des particuliers.» $\mathrm{Si}$ «à leur époque de fécondité, les Français ne se sont pas montrés à cet égard inférieurs aux autres races», quelle est l'explication de l'inaptitude actuelle des Normands, par exemple, à coloniser? «C'est la dure contrainte imposée depuis 1793 à la race normande ...; c'est la substitution du Partage forcé à la coutume qui y maintenait la transmission intégrale des biens et la fécondité des mariages.») Si Le Play a influencé Rameau, il n'est pas impossible qu'en retour celui-ci lui ait fourni des arguments, comme le laisserait croire cet autre extrait du sociologue: «Pour retrouver les traces de la fécondité qui distinguait les familles normandes avant la Révolution, il faut maintenant visiter les essaims du Canada. Les colons normands du Bas-Canada ont conservé à un degré remarquable la force d'expansion. Sous ces deux rapports ils l'emportent même sur les Anglo-Saxons qui défrichent concurremment avec eux le bassin du Saint-Laurent.» (Notons en passant comme la doctrine leplaysienne peut justifier une interprétation optimiste de l'émigration aux États-Unis.) Les citations ci-dessus sont tirées de «La colonisation», dans la revue L a Réforme sociale, Se série, 5 (1903): 885-88. 
Rameau et de quelques-uns de ses confrères, elle occupera une certaine place dans les relations franco-québécoises: à Paris, en accueillant fréquemment des Québécois de marque, tels le curé Labelle ou le premier ministre Mercier; ici, en suscitant la formation de groupes leplaysiens. Dans les années 1890, Rameau pourra se dire l'ami de Levasseur ${ }^{8}$. À l'époque du concours, il est douteux qu'il en ait été ainsi. De toute façon, les oeuvres étaient inscrites sous le sceau de l'anonymat, ce qui confère aux commentaires du jury une valeur particulière.

On présentera ici une analyse du travail de Rameau en insistant sur les parties qui intéressent l'Amérique française ou qui véhiculent des images de l'autre Amérique: l'Amérique anglosaxonne, l'Amérique victorieuse. Il est évident que, sans la participation de Rameau, le degré d'attention accordé à l'expérience canadienne-française dans ce concours aurait été plus modeste, les États-Unis étant le point de mire ${ }^{9}$. C'est par cette insistance que l'oeuvre de Rameau en général et ce mémoire en particulier prennent place de plein droit dans notre production culturelle savante au XIXe siècle. Ces écrits ont aussi valeur de témoignage sur l'esprit qui animait la plupart des «amis du Canada» en France.

Les quelque 700 pages du mémoire de Rameau se divisent en deux grandes parties d'inégale longueur comportant au total qua-

8 ANQ (Québec), fonds Rameau, brouillon d'une lettre à Mgr Hamel, 26 mai 1893; fonds Le Sage, Rameau à S. Le Sage, 14 mai 1894. Archives du Séminaire de Québec, fonds Verreau, Mme Rameau à l'abbé Verreau, 26 mai 1893. Les archives Decencière Ferrandière renferment cinq lettres de Levasseur à Rameau, de 1889 à 1896. On doit à Levasseur (1828-1911), membre de l'Institut, professeur au Collège de France et au Conservatoire des Arts et Métiers, des travaux importants sur la colonisation française. Citons la France et ses colonies (géographie et statistiques) (Paris, 1890-93). Il est question du Canada dans le tome 3. Dans ses travaux, il s'est aussi intéressé au système québécois d'instruction publique. Ses oeuvres les plus connues restent son Histoire des classes ouvrières et de l'industrie en France avant 1789 (Paris, 2e éd., 1900-01) et son Histoire des classes ouvrières et de l'industrie en France de 1789 à 1870 (Paris, 2e éd., 1903-04). Des divergences de vues existaient entre Rameau et Levasseur malgré leurs relations cordiales. «J'aurais peut-être, écrit Levasseur, quelques réserves d'opinion à faire sur la manière dont vous jugez l'état social de la France dans les siècles passés» (lettre à Rameau, au sujet d'Une colonie féodale, 28 juill. 1889 , dans les archives Decencière Ferrandière). Désaccord sur le plan historique ou idéologique, mais aussi sur le plan philosophique ou religieux, d'où cette mise en garde de Mme Rameau à l'abbé Verreau: Levasseur «sans y être hostile ne partage assurément pas toutes vos croyances chrétiennes» (26 mai 1893). Un "ami du Canada» sans doute, mais dont le signalement ne correspond pas au type habituel, conservateur et catholique, sinon ultramontain.

$9 \quad$ Rameau n'est pas étranger au mouvement des idées au Québec, mais l'influence qu'il a pu y exercer n'est rien - toute proportion gardée - à côté de celle du Canada français sur sa propre évolution intellectuelle et l'orientation de son activité et de ses travaux. Il en était conscient comme le révèle cette confidence, peu avant sa mort, à son vieil ami Le Sage: «... ce charmant pays [le Canada], que j'ai tant admiré, et tant aimé, et dont l'étude a été pour moi une révélation salutaire; salutaire non pas seulement pour moi, mais aussi pour plusieurs autres, comme on pourra le voir, dans la suite, je crois, pour plusieurs autres!»» (ANQ (Québec), fonds Le Sage, Rameau à S. Le Sage, 3 sept. 1899.) 
torze chapitres. La seconde, d'environ 135 pages, examine la colonisation au XIXe siècle. Quant aux 500 pages de la première partie, elles étudient les diverses colonisations européennes à l'époque moderne. Cette affirmation appelle des réserves importantes. D'abord la part du lion va au XVIIe siècle (au XVIe dans le cas de l'Amérique espagnole), c'est-à-dire aux origines des colonies, «parce que, précise l'auteur, nous avons cru y voir le fait capital, qui domine tout le reste de leur développement» (préface). Par la suite, dans son oeuvre historique, Rameau restera fidèle à cette prédilection. C'est ainsi que son grand ouvrage sur l'Acadie s'intitulera Une colonie féodale (2e éd., Paris, 1889), bien que ce titre ne convienne vraiment qu'aux toutes premières décennies, et encore à la condition d'admettre la synonymie avec seigneurial et de n'y pas regarder de trop près. Ainsi que l'observait Benjamin Sulte, même avec cette atténuation, «le titre contredit le livre» ${ }^{10}$. Et Sulte d'ajouter: Rameau «ne rencontre pas de colonie féodale, toutefois il lui semble qu'elle est dans l'air. Le seigneur hante son imagination». C'est assez justement trouvé. En effet, avec l'école historique française dite catholique, Rameau en viendra à concevoir la féodalité sous une forme idéalisée, comme état social et comme univers culturel. Il lui semblera redécouvrir en Acadie et au Canada le type «féodal» de relations entre la famille censitaire et son petit patrimoine foncier, la même constellation de croyances, de principes et de moeurs. Il expliquera la survie et les progrès, en dépit de la conquête, des colons acadiens et canadiens par la «féodalité qui était dans l'air» à l'origine et pour laquelle leur attachement ne se démentira pas ${ }^{11}$. Rameau privilégie donc, comme explication, la raison culturelle un peu comme Fustel de Coulanges rend compte de la Cité antique par la religion antique. Les débuts constituent le «fait capital» parce qu'alors sont mises en place les durables structures mentales et morales qui informeront les développements dans la suite des temps. Telle est l'idée directrice, fondamentale de l'ouvrage, et qui en règle l'économie.

Pour nous nous reconnaissons sans hésiter, qu'au-dessous de toutes les mécaniques sociales, nous trouvons partout, dans la grande étude de l'histoire, et dans l'observation journalière et commune du Présent, une force autrement importante, autre-

10 B. Sulte, «Critique sans malice», Revue acadienne, 2 (1918): 21-22. Rappelons, pour donner la mesure de la liberté prise par Rameau avec le vocabulaire, que Guy Frégault, dans la première édition de la Civilisation de la Nouvelle-France, 1713-1744, avait coiffé le chapitre quatre du titre «Une colonie féodale». Dans la réédition chez Fides, en 1969 , on lit plutôt «Sous le régime seigneurial».

1 Que le lecteur nous permette de le renvoyer, sans plus, aux deux articles suivants: Pierre et Lise Trépanier, «Rameau de Saint-Père et le métier d'historien», RHAF, 33,3 (décembre 1979): 331-355; «Rameau de Saint-Père et l'histoire de la colonisation française en Amérique», Acadiensis, 9 (1980): 40-55. 
ment dominatrice, c'est celle des doctrines qui forment nos convictions et nos pensées. Cette force des idées a joué dans le monde un rôle supérieur à celui de toutes les combinaisons politiques et sociales, et nulle part je pense elle n'a mieux montré sa puissance et son efficacité que dans la formation de ces grandes colonies que nous venons d'étudier (686-87)!2

Notre historiographie prolongera cette logique des origines, présente aussi mutatis mutandis chez Garneau et Ferland. La vision de la Nouvelle-France du chanoine Groulx en reçoit son inspiration centrale. Au-delà de la défaite, le Canada français porte en lui des promesses de réalisation parce qu'il est «né, peut-on dire, de la meilleure France» et que «sa parfaite homogénéité ethnique et religieuse» est garante de sa fidélité à ses origines ${ }^{13}$.

Mais, pour Rameau, ces «doctrines» ne se cristallisent pas dans la cornue des alchimistes de la pensée. Parce qu'artificiels, les systèmes des hommes de cabinet sont mort-nés, à preuve les projets de Locke, Penn ou Oglethorpe. Les «doctrines» vivantes qui animent les civilisations s'élaborent obscurément dans les profondeurs de la tradition. Elles sont reçues, non pas inventées:

Les Doctrines religieuses ou morales qui présidèrent à l'établissement de ces colonies, parurent au contraire dès l'abord composées de toutes pièces, telles qu'elles devaient demeurer ensuite; elles ne se sont point modelées sur la société en progression, mais ce sont les sociétés dans leur progression qui se sont modelées sur elles (689).

Et dans l'optique de Rameau, l'idée féconde à la base de toute doctrine, l'idée mère, le soleil au centre de tout système culturel, c'est la religion.

La thèse principale qu'on vient d'évoquer corrobore le découpage qu'ailleurs nous avons opéré dans l'évolution intellectuelle de Rameau: jusqu'en 1852, c'est un catholique révolutionnaire, fervent partisan de 1848 , pour qui le salut de la société française passe par la République car il croit à la toute-puissance des institutions politiques; de 1853 à 1865 , il vit une période de transition dont l'orientation est renforcée par le traumatisme de 1870-71; puis, jusqu'à sa mort, il professe un traditionalisme catholique de plus

12 Les chiffres entre parenthèses à la suite d'une citation ou d'un résumé correspondent à la pagination du mémoire manuscrit de Rameau (Du système...).

13 L. Groulx, Histoire du Canada français depuis la découverte, 4 éd., 1: 17. D'une certaine manière, l'explication par les origines est inhérente à toute approche historique, idéaliste ou non. Des théoriciens des sciences sociales y ont aussi recours. Qui ne connaît la théorie du fragment de Louis Hartz? André-J. Bélanger s'en inspire tout en émettant des réserves dans l'Apolitisme des idéologies québécoises (Québec, 1974). Voir la brève mais suggestive mise au point sur Hartz, Lipset, Almond et Mannheim dans D. Bell et L. Tepperman, «Perspectives on political culture», The Roots of Disunity (Toronto, 1979), 11-39. 
en plus doctrinaire, de moins en moins sensible aux caprices du réel dans son irréductible foisonnement. Or cette primauté des croyances et des moeurs, la pertinence toujours actuelle des traditions qui ont donné à l'Occident sa force, sa grandeur et ses progrès, la Science sociale de Le Play les redécouvre empiriquement. Les travaux de l'école édifient un positivisme de la tradition qui ne fait pas mystère de ses prétentions scientifiques. Rameau reconnaît sa famille idéologique et adhère, en 1865 , à la Société d'économie sociale. Cette répudiation du personnage radical, qui était l'une des composantes de sa personnalité complexe, lui a valu la place d'honneur qu'il a occupée dans le monde intellectuel québécois de la deuxième moitié du XIXe siècle. Si au lieu d'évoluer vers le conservatisme, il avait exagéré ses tendances démocratiques et socialisantes - même en intensifiant l'effort de conciliation avec le catholicisme de son temps, - il aurait vu son audience au Canada français se rétrécir comme peau de chagrin. Pour s'en convaincre, on n'a qu'à rappeler l'accueil réservé à la France aux colonies par notre élite dominante. Flattée de la thèse de la supériorité du colon français, exaltée par la mission intellectuelle et morale que le livre assignait au Canada français, elle ne s'est guère attardée au libéralisme, pourtant l'un des fils conducteurs de l'ouvrage, sauf à y voir la confirmation de l'excellence des institutions parlementaires, l'une des assises de son pouvoir social.

Dans le mémoire de Rameau, l'étude des diverses colonisations traduit une préférence dans le temps, comme on l'a vu, mais aussi dans l'espace. L'auteur avoue avoir «négligé les colonies intertropicales à esclaves, qui semblent plutôt des Ateliers de Fabrication, artificiellement établis à l'aide de l'esclavage, plutôt que de véritables colonies» (préface). L'escamotage est de taille: toutes les îles à sucre, joyaux pourtant des domaines coloniaux aux yeux de l'homme des XVIIe et XVIIIe siècles. Mais là n'est pas le point de vue de Rameau, qui, fidèle à sa vision idéaliste des choses et de l'histoire, a une tout autre conception de la colonisation:

Toute colonisation sérieuse nous a paru impliquer soit la transformation des populations préexistantes, ce qui était le caractère général des colonies antiques, soit la transplantation d'une population nouvelle, ce qui est le trait le plus commun parmi nos colonies modernes (préface).

L'auteur appelle colonie d'importation un établissement colonial constitué par transplantation de population. Il ne cache pas que la colonie d'importation lui semble de beaucoup inférieure à la colonie par transformation. Les peuples modernes, même éclairés par la religion chrétienne, n'ont pas su imiter en cela les Anciens. Con- 
trairement à ces derniers, ils manifestent une impatience maladive dans les créations sociales (105-106), oublieux qu'ils sont de l'incapacité de la loi à perfectionner les sociétés (36-37): les constitutions ne font pas les peuples (39). Les peuples sont le fruit du lent cheminement des forces morales, responsables à travers la tradition des véritables progrès: perfectionnement de la vie familiale, développement personnel, responsabilité individuelle (95-96). Le législateur ne peut décréter ces progrès moraux, l'histoire des révolutions de la fin du XVIIIe et de la première moitié du XIXe siècle le prouve assez. Bien des illusions se sont dissipées: "Quand on imagine que la liberté est un principe absolument fécond et bienfaisant, on procède comme ces charlatans qui font d'un certain remède une panacée universelle» (54-55). Si les institutions politiques et la loi se montrent plutôt impuissantes dans l'ordre des forces morales, évidemment il en va tout autrement de la religion. Pourquoi les peuples modernes n'ont-il pas mieux suivi les enseignements du christianisme qui croit si fermement à la transformation de toutes les branches de la famille humaine? Pourquoi, plutôt que de violenter le temps, ne se sont-ils pas associés à son oeuvre? C'est qu'ils ont trop souvent conçu la colonisation à travers les catégories étroites du mercantilisme et du gain à court terme. Ils ne la voyaient pas assez comme une «expansion civilisatrice» (444). Rameau s'élève contre cette erreur:

Nous voulons bien être des producteurs mais nous ne consentons pas à être des éducateurs; nous n'admettons pas de peuples enfants en communion avec nos travaux, nous ne tolérons dans la sphère civilisée que des peuples d'hommes faits (445)!

En effet, le système colonial de l'auteur repose sur le refus du racisme (65-67) et de son corollaire, l'esclavage, "phénomène monstrueux et menaçant, problème honteux» (53).

Rameau n'est pas raciste en ce sens que les inégalités entre les peuples ne lui paraissent pas avoir le caractère de permanence nécessaire que leur conférerait une détermination biologique inscrite à tout jamais dans la race. Tout peuple est perfectible, en tout cas «il est impossible de préciser quelles sont les races humaines utiles ou inutiles à conserver» (71). Il faut donc condamner les écrivains qui «ont dénoncé les indigènes de l'Amérique comme une population uniquement propre à être tenue constamment en tutelle, une superfétation de l'humanité, nécessairement appelée à disparaître» (66). Le développement des peuples est fonction de plusieurs facteurs, dont le réseau des influences exogènes et le temps: «Dans le développement de la civilisation, le temps est un élément qui ne peut se suppléer; on ne peut le méconnaître sans 
tout compromettre, et il faut nécessairement lui accorder sa part» (71). De là découle une seule position acceptable: «C'était ainsi satisfaire à la fois la justice et l'intérêt bien entendu de l'humanité que d'assurer le droit à l'avenir à toutes les familles humaines»" (71). Le catholicisme n'a pas compris autrement sa mission parmi les nations.

À l'époque moderne - toujours selon Rameau, - il n'y a guère eu que la colonisation espagnole à se rapprocher de la colonisation par transformation, et encore participe-t-elle d'un type ambigu, hybride. Ce qu'elle a eu de bon doit être attribué «point tant au génie espagnol qu'au génie du catholicisme» et à l'influence modératrice de l'autorité royale (48). «En deux mots la conséquence directe de la politique espagnole et de l'éducation familière, doucement progressive, donnée par le clergé, fut la consécration définitive de la race indienne consolidée» (53). Par conséquent, on ne saurait trop louer l'oeuvre des missionnaires, même quand leur zèle imprudent les a amenés à la formule discutable des réductions. Les colonies espagnoles ont cependant eu le malheur de recevoir une immigration douteuse, d'assister au déclin moral de leur clergé et d'avoir à tenter leur développement sous un régime de cueillette des produits monnayables plutôt que de mise en valeur du pays. Les conséquences de ce système ont été tragiques pour les colonies; les répercussions, terribles pour la métropole, qui, croyant s'enrichir, s'appauvrissait: «Il se vérifia une fois de plus que la richesse sans le travail qui est son titre légitime, et sans l'activité qui la vivifie, perd toute vertu; bien plus elle devient funeste!» (113). Le pillage des colonies à la faveur de l'exclusif colonial aboutit à la ruine et de la métropole et des colonies. «L'Espagne en fit la triste expérience, et cette ombre de profit qu'elle tirait sur l'heure de l'Amérique, étaient (sic) véritablement les entrailles de la Poule aux Eufs (sic) d'or» (120). En résumé, tel était le propos des quatre premiers chapitres de l'ouvrage, consacrés à la colonisation espagnole.

Suivent sept chapitres traitant des colonies d'importation au XVIIe siècle: un sur l'Acadie, deux sur le Canada avec quelques pages sur la Louisiane, trois sur les colonies anglo-américaines y compris la colonie de la Nouvelle-Hollande, enfin un portant sur la colonisation du Cap de Bonne-Espérance par les Pays-Bas.

Aux yeux de Rameau, de toutes les colonisations en terre d'Amérique, la plus noble, par ses motivations, a été la française: «Les Espagnols se précipitaient vers le Nouveau-Monde enfiévrés par l'appât des mines et des richesses; les Anglais s'établirent aux États-Unis par fanatisme religieux, ou sous les nécessités de la persécution politique. Ces colonisateurs français nous présentent seuls l'idée chevaleresque de la patrie à étendre et de l'humanité à 
développer» (144). Nous avons exposé ailleurs les thèses de Rameau sur la colonisation française en Amérique. Qu'il suffise de signaler ici que le mémoire se présente, à certains égards, comme une transition de plus dans l'évolution de l'historien et de l'idéologue, à partir de la France aux colonies, en 1859, jusqu'à Une colonie féodale (1 re édition en 1877, 2e édition revue et augmentée en 1889). On le voit, par exemple, dans l'examen de la liberté comme facteur de réussite des colonies. Comparant les établissements de Beauport, de Montréal et du Massachusetts, Rameau conclut dans son mémoire qu'on a exagéré le rôle de la liberté et qu'il n'est «point aussi prouvé que l'on pourrait le croire, au moins dans les faits que nous étudions, que le libre essor du travail spontané soit de beaucoup supérieur au travail discipliné dans l'établissement d'une colonie» (191). Si la colonie de Montréal et les colonies puritaines ont en commun la discipline, elles partagent aussi ce caractère spécial de faire procéder l'ordre de l'idée religieuse: «Il peut se faire en effet que la discipline volontairement délibérée dans l'esprit qui la subit, soit supérieure à la liberté, comme celle-ci paraît être supérieure à la discipline aveuglément subie» (192). On pressent comment peu à peu l'idée religieuse va orienter la réflexion du démocrate de 1848 dans le sens d'une banalisation de l'idée de liberté ${ }^{14}$. Mais pour l'heure l'historien continue à s'appesantir, comme en 1859, sur l'absence néfaste de système municipal en Nouvelle-France: "L'institution municipale au Canada aurait dû être la pierre angulaire de la colonisation» (262). L'auteur mettra toutefois une sourdine à cet argument en 1877, pour l'évacuer en 1889. D'ailleurs, plus que les institutions, ce qui importe pour le développement des colonies de transplantation, c'est la qualité et la quantité des premiers contingents de colons, puis leur accroissement naturel (158). Encore là, la religion joue un rôle essentiel: elle est garante de l'honnêteté des moeurs et, partant, de la fécondité des familles. De toutes les supériorités que les auteurs attribuent aux colonies américaines sur la Nouvelle-France, une seule au fond a été déterminante: la forte immigration qui a conféré aux colonies américaines, dès le XVIIe siècle, un avantage numérique écrasant. On comprend aisément que cette insistance sur la religion, la moralité et le taux de natalité fournit à l'auteur un argument historique à ses yeux irréfutable en faveur de la colonisation française

14 Liberté politique surtout, car en bon leplaysien Rameau souhaite, pour le père de famille, la plus large mesure possible de liberté économique et testamentaire. Pour le père de famille puisque, dans la doctrine de Le Play, la vraie unité sociale, c'est la famille plutôt que l'individu. 
en Algérie et canadienne-française en Amérique: le Français est un excellent colon ${ }^{15}$.

Les chapitres sur les colonies anglo-américaines sont les plus intéressants. Ils aident à mettre en perspective l'image que se faisait Rameau des États-Unis du milieu du XIXe siècle. On ne peut douter de la pertinence d'une reconstitution nuancée de cette image car elle est à la base du discours et de l'action de Rameau en Amérique. Ainsi elle permet de mieux saisir les justifications de son combat - de concert avec le curé Labelle, l'abbé Proulx et Mgr Racine au Québec, Pascal Poirier en Acadie et le curé Chagnon chez les Franco-Américains - contre la suprématie que prétendaient exercer les Irlandais dans l'Église catholique d'Amérique du Nord. Elle éclaire aussi l'antiaméricanisme de la plupart des intellectuels québécois (mis à part les Rouges et leurs épigones): sur ce point comme sur les autres, l'influence de Rameau a pu se déployer dans l'exacte mesure où sa pensée se rencontrait avec celle de l'idéologie dominante.

$\mathrm{Au}$ point de vue historique, Rameau contredit les interprétations à l'honneur à son époque, qui opposaient la Nouvelle-France féodale aux colonies américaines libres et démocratiques. Pour lui, la Virginie, le Maryland, les Carolines étaient des colonies seigneuriales. La ressemblance avec la Nouvelle-France lui paraît digne d'être relevée. Même les colonies puritaines, à son avis, "avaient été constituées sous ce mode seigneurial» (377). Il s'attarde au cas de la Nouvelle-Angleterre parce que l'esprit yankee est celui qui prédomine dans les États-Unis de son temps. Or que révèle l'étude des fondateurs des établissements puritains? «Ils n'étaient ni despotes, ni démocrates, peut-être étaient-ils un peu républicains par nature, mais ils étaient surtout seigneurs-actionnaires, et puritains fanatiques; comme tels ils usaient de leurs droits seigneuriaux dans l'intérêt de leur secte, comme d'autres en usaient dans leur intérêt personnel (...) c'étaient des seigneurs par action» (380). Rameau restera fidèle à cette idée que le mode de colonisation seigneuriale était courant en Amérique. N'écrira-t-il pas dans l'édition de 1889 d'Une colonie féodale: «Toutes les colonies du nord de l'Amérique furent ainsi établies sur le même plan, avec certaines variations

15 La logique de l'idée religieuse ainsi que de la simplicité et de la fécondité des familles fonde aussi les admonestations de Rameau qui exhorte le Canada français à rester fidèle à sa foi, à ses traditions, et docile à son clergé. Si, dans les dernières années de sa vie, Rameau a paru désespérer du Canada français, c'est précisément parce qu'il interprétait certains signes, comme l'arrivée des libéraux au pouvoir tant à Ottawa qu'à Québec, comme le début d'une rupture avec la tradition, une plus grande perméabilité à l'américanisation et une résurgence de l'anticléricalisme. 
dans leurs institutions, variations qui n'avaient peut-être pas toute l'importance qu'on leur a attribuée depuis lors. ${ }^{16}$

Il savait bien qu'il s'agissait, surtout pour la NouvelleAngleterre, d'une généralisation quelque peu téméraire. Il l'a avoué à Beamish Murdoch, qu'il appelait en renfort ${ }^{17}$. Généralisation téméraire, mais utile à la démonstration: sous-estimer les divergences institutionnelles et les autres contrastes entre les deux sociétés coloniales permet de mieux isoler l'avantage numérique comme explication, en dernière analyse, de la chute de la NouvelleFrance, de réaffirmer la valeur du colon français et de vérifier l'idéologie leplaysienne, qu'embrasse Rameau au cours de ces années: religion, famille, patrimoine foncier et hiérarchie sociale sont la clé du bonheur des peuples. La «féodalité», réhabilitée, devient un système progressiste. Par rapport à 1859 , on constate quelle rupture marque cette insistance sur le caractère féodal des colonies anglo-américaines. L'ancien théoricien politique concède l'infériorité de la raison face à l'expérience. Ses invitations à l'humilité dévoilent la crise de conscience qu'il vient de vivre (518-19).

Rameau admire les colons puritains pour leur valeur morale et leurs convictions religieuses, bien que ces dernières n'aillent pas sans une certaine étroitesse d'esprit. Il loue leurs talents d'hommes d'affaires, se demandant «quelle secrète relation il peut exister entre ces aptitudes commerciales et la doctrine des Puritains» (393). Mais il rejette le mythe du Puritain père de la liberté. Sa conception est plus nuancée:

En aucune autre colonie l'usage des libertés politiques n'était aussi habituel, aussi répandu, et nulle part cependant on ne rencontrait une telle rigueur d'inquisition, un tel déni de la liberté de pensée! C'est ainsi que par une de ces singularités propres à l'esprit humain, si merveilleusement souple dans ses évolutions, la Nouvelle-Angleterre allia une extrême intolérance dans les sphères élevées de l'intelligence, avec un développement très sérieux de la liberté, dans les régions de la politique et des intérêts privés; et c'est de cette situation particulière qu'est dérivé cet esprit libéral et très étroit à la fois, ingénieux et médiocre, subtil mais dépourvu d'ampleur, qui caractérise encore aujourd'huy la population yankee $(381 \mathrm{~b})$.

Il corrige les observateurs qui se sont mépris sur les véritables talents et défauts des Puritains. Comme colons, on leur a prêté une audace et un dynamisme qu'ils n'avaient pas alors qu'en Nouvellelon).

6 Rameau, Une colonie féodale (Paris, 1889), 1: xviii.

17 ANQ (Québec), fonds Rameau, Rameau à B. Murdoch, 10 août 1877 (brouil- 
France on laisse dans l'ombre les mêmes qualités, qui pourtant y surabondaient.

Rien de moins audacieux, de moins énergique et de plus casanier que le Yankee primitif, il était habile et entreprenant dans les affaires commerciales, mais point dans la colonisation, non plus que dans la guerre; ce n'est que par force de monde, et en se poussant de proche en proche qu'ils gagnèrent du terrain et pénétrèrent dans le continent. Ces allures conquérantes, et cette effronterie d'audace qu'ils affectent aujourd'huy leur sont venues fort tard avec l'habitude du succès; mais ces traits ne leur sont point naturels, et c'est pourquoi sous cette nouvelle figure, ils offrent toujours un certain caractère de vanité vantarde, mesquine, et même légèrement impertinente; c'est que toute cette assurance leur vient plus des faveurs de la fortune que de leur propre coeur (503).

Ainsi donc, de graves menaces pèsent sur les États-Unis contemporains. Les qualités qu'avec le temps ils ont acquises mettent en péril celles qui constituaient le fond de leur caractère primitif. Leur réussite matérielle compromet leur valeur morale: ils n'ont pas su concilier et faire marcher de pair le progrès matériel et le progrès spirituel. Leur société étale un matérialisme qui présage peut-être la décadence. Inférieurs comme colons aux Canadiens du XVIIe siècle, les Américains ont édifié, au XIXe siècle, un type inférieur de civilisation ${ }^{18}$. La société canadienne-française, moins riche il est vrai, a néanmoins progressé démographiquement et économiquement, tout en préservant sa valeur primitive et sa moralité. Ainsi se trouve démontrée la supériorité du Canada français dans le passé et dans le présent, et fondée sa mission religieuse et civilisatrice. Malgré les apparences, c'est à lui que la destinée sourit.

Trois chapitres forment la seconde partie qui, on se le rappelle, porte sur la colonisation au XIXe siècle. Le chapitre sur les colonies anglaises de l'Amérique du Nord montre que les Acadiens

18 Léon Gérin, leplaysien mais de la branche tourvillienne, prendra le contrepied des thèses de plus en plus catégoriques de Rameau: «L'étude comparée des progrès de la colonisation au Canada et aux États-Unis, écrit le jeune sociologue, me fait voir d'une manière bien saisissante la différence de l'Angleterre et de la France dès le dix-septième siècle. Que nous nous transportions sur les grandes plantations des États du sud ou sur les domaines plus restreints des petits patrons de la Nouvelle-Angleterre [...] partout et toujours se manifeste d'une manière extraordinairement vigoureuse et pratique l'action d'une classe supérieure agricole. Contraste affligeant pour nous avec la Nouvelle-France où nous voyons une race de paysans, excellente pourtant, languir pendant des siècles faute de chefs naturels. Chaque fois qu'il se présente quelque intérêt tant soit peu compliqué, l'habitant est incapable d'agir et jette les hauts cris. Et qui trouve-t-il pour le secourir? Autrefois c'étaient des fonctionnaires titrés, gentilhommes pauvres et par nécessité soldats avant tout. Triste peinture. Aujourd'hui c'est un clergé, généralement peu éclairé et des politiciens»» (Saint-Jérôme, Archives des Jésuites, fonds Gérin, L. Gérin à H. de Tourville, 20 août 1890 [copie]). 
et les Canadiens sont restés tels qu'en eux-mêmes. Le fait central est «cette conquête pacifique, opérée en sous-oeuvre par le peuple vaincu sur le peuple vainqueur», c'est-à-dire l'expansion démographique, qui par exemple, se traduit par la reconquête des Cantons de l'Est (557). Le chapitre sur les colonies britanniques d'Afrique du Sud et d'Australie corroborera, par l'observation contemporaine, les enseignements de l'histoire: "Ici encore nous allons retrouver les mêmes maladresses, les mêmes fautes, les mêmes défaillances qui caractérisent invariablement les débuts de toutes les colonies anglaises» (590). Le dernier chapitre - sur l'Algérie établit un bilan de la colonisation française, qui, dans ces contrées, selon Rameau, n'a vraiment commencé qu'en 1841 (600). Or la colonie algérienne, malgré ses misères et ses mécomptes, est pleine de promesses et, moyennant les correctifs indispensables, la France pourra enfin y réaliser l'oeuvre colonisatrice digne d'elle et de ses colons.

Dans les «Considérations générales» (661-97), l'auteur ramasse en faisceaux les grandes idées qu'il a voulu défendre. Si son anglophobie le pousse à fustiger les anglomanes, «adorateurs du succès» (665), elle ne l'aveugle pas sur la suprématie coloniale de la Grande-Bretagne au milieu du XIXe siècle et l'essor étonnant des États-Unis. Deux ordres de préoccupations agitent ses pensées: les dangers que comporte la situation pour les Canadiens français; la raison profonde de l'évolution historique qu'il a décrite. La France a perdu son empire d'Amérique parce qu'un concours de circonstances l'a privée des immigrants qui, en revanche, affluaient dans les colonies rivales. Soit. Mais l'esprit de l'historien reste insatisfait de cette explication, qui ne fait qu'aiguillonner sa réflexion.

Quant au destin des Canadiens français, une inquiétude s'insinue dans la belle assurance de l'idéologue. (Notons dès maintenant que cette inquiétude paraît avoir décru par la suite, en proportion du renforcement du traditionalisme chez notre intellectuel, pour ne reparaître qu'à la fin de ses jours, mais amplifiée jusqu'à l'angoisse.) Rameau se rend compte que les Canadiens français ont surtout produit des hommes, et les Américains, de la richesse. Plus nombreux de seulement la moitié, les premiers, supérieurs par l'audace et l'esprit de ressources, auraient été en mesure de conquérir 
les colonies américaines ${ }^{19}$. Le tempérament bien trempé du Canadien français comporte toutefois une faiblesse: s'il est ardent à l'ouvrage, il se contente de peu et son ambition est bornée. Il découlait de la mentalité et du milieu socio-politique des colons de la Nouvelle-France que «leur intelligence qui ne s'était jamais élevée jusqu'aux affaires publiques, ni jusqu'aux idées générales, n'était point tourmentée par la recherche de progrès qu'elle concevait fort peu» (676). Cet idéal social aimable ne va donc pas sans risque:

Peut-être pour le simple bonheur des hommes et la force durable des sociétés, cette modération sage et honnête des désirs vaut-elle mieux dans une raisonnable activité! Mais au milieu de la concurrence générale des peuples, cela pourrait conduire à un amoindrissement relatif ${ }^{20}$; car l'activité industrieuse restant moindre, il arrive promptement que les ressources d'une telle société se trouvent trop inférieures à celles des autres (676).

Le voisinage des Anglo-Canadiens et surtout des Américains recèle bien des périls, que l'idéologue conjurera de mieux en mieux, pour la paix de son esprit, au fur et à mesure qu'il étoffera sa symptomatologie de la décadence américaine. Mais il n'empêche que persiste la possibilité d'une répétition du scandale qui le trouble tant dans l'histoire de l'Amérique du Nord: les meilleurs ont eu en partage la défaite.

Une «fatalité heureuse» a fait de la Grande-Bretagne un des plus grands foyers d'émigration européenne au XVIIe siècle (69596). Â l'avantage de la quantité, s'est joint - au moins dans le cas des Puritains - celui de la qualité: des familles choisies, qu'animent «des principes moraux appuyés sur des croyances religieuses» (693). Mais la conjonction de crises religieuses et de troubles politiques en métropole n'épuise pas l'explication de ce fait surprenant

19 Cette opinion, qui se trouve à la page 663 , et aussi celle de la page 144 sont empruntées à F.-X. Garneau, Histoire du Canada, 2e éd., 1852, 1: 300-01. Rameau n'a pas inventé une interprétation traditionaliste et ruraliste de notre passé; il l'a plutôt découverte chez nos historiens, Garneau et Ferland, pour nous la resservir apprêtée à sa façon. Reprises par Rameau, ces idées n'ont d'importance, dans notre histoire intellectuelle, que parce qu'elles sortaient cette fois de la plume d'un étranger, qui de la sorte les confirmait en leur donnant un certain lustre. Ainsi dans leur Histoire du Canada (2e éd., 1919), les Frères des Écoles chrétiennes se réfèrent souvent à Rameau, dont ils admirent la «manière véridique et brillante» (518). Plus loin (622), le voyageur français sert de caution à notre pureté culturelle: «Sur les bords du Saint-Laurent, observe Rameau, notre langue n'a pas plus dégénéré que notre caractère.» Telles étaient les utilisations idéologiques de Rameau.

${ }_{20}$ La même inquiétude n'accompagnait pas l'antiaméricanisme déjà présent en 1859 ou, en tout cas, n'était pas aussi apparente. Il y avait pourtant un avertissement: ne pas «négliger le nécessaire; on peut, comme nous le faisons en France, s'adonner aux sciences et aux beaux-arts, et cependant entretenir un mouvement d'industrie et de commerce proportionné à l'importance de son pays». La France aux colonies (Paris, 1859), 267. 
que les Anglo-Saxons, dans la lutte pour la domination des Amériques, ont surpassé les Espagnols malgré la solide avance de ceux-ci dans le temps et l'opulence de leurs possessions, et évincé les Français en dépit de la supériorité de ces derniers comme colons. L'historien contemple ce réseau «de merveilleux hazards (sic), de concordances extraordinaires qui toutes concourent au but final, à la consolidation et au développement de la race anglo-saxonne, et à la ruine de ses rivaux» (697), - et se dit: «Il y a là autre chose que le vouloir et la main de l'homme» (694). Ici l'histoire s'assimile à la théologie de l'histoire:

C'est là sans contredit un des faits historiques où se montre de la manière la plus surprenante cette force supérieure qui gouverne l'ensemble des hommes, sans préjudicier à la liberté de l'homme; ce caractère providentiel que tant d'esprits éminents se sont plu à rechercher dans l'Histoire se révèle ici avec de singulières clartés; car on ne trouve nulle part au même degré, tant de coïncidences capitales et imprévues, tirant le succès de l'inhabileté, précipitant la ruine malgré le courage, défiant la Victoire elle-même, et faisant sortir le Oui du Non avec une puissance persistante, indiscutable, indépendante de toute sagesse et de tout calcul humain (697).

Voilà pourquoi il convient de chercher les signes et de surprendre leur secret, même si l'anxiété étreint le coeur. D'où la rassurante fonction du providentialisme et de son prolongement, le messianisme. La religion pénétrant et soutenant toute la réflexion, on ne s'étonne pas de voir le penseur quitter le plan des choses humaines, des choix politiques, des responsabilités conscientes pour accéder au plan mystérieux mais prégnant de la transcendance.

Comment le jury et, en particulier le rapporteur, Émile Levasseur, ont-ils réagi au mémoire de Rameau? Le mémoire a été classé troisième. On se souvient que la première place est allée au travail de Leroy-Beaulieu ${ }^{21}$. D'après Levasseur, ce dernier ouvrage est le seul à avoir embrassé toute la question: "Il a bien envisagé le problème sous sa double face: influence des divers régimes sur le développement des colonies, et influence des colonies elles-mêmes sur les métropoles.» ${ }^{22}$ La typologie retenue est aussi plus exhaustive

21 Alexis Delaire a publié un compte rendu de l'étude de Leroy-Beaulieu, édition de 1882, dans la Réforme sociale, 2e année, t. 2 (1882): 108-15, sous le titre: «La France et la colonisation, À propos d'un livre récent». Cet article très élogieux montre - encore une fois - que la Société d'économie sociale favorisait le mouvement colonial. Il révèle aussi que, dans ce milieu, on considérait Rameau comme une autorité sur les questions coloniales nord-américaines: par deux fois, Delaire fait référence à ses travaux. Sylva Clapin a donné un compte rendu de la 4e édition dans la Presse, 28 nov. 1891, 6.

22 E. Levasseur, «Rapport sur le concours pour le prix fondé par Mme Léon Faucher», Mémoires de l'Académie des Sciences morales et politiques, 13 (1870): 508. 
que celle de Rameau ${ }^{23}$ : colonies de commerce (les simples comptoirs, par exemple Singapour ou Hong-Kong); colonies de plantations (établissements tropicaux, telles les Antilles, fournissant des denrées d'exportation exotiques); colonies agricoles (comme les États-Unis, l'Australie ou le Canada). Ces dernières apparaissent comme des projections de la métropole qui préparent «pour l'avenir des peuples faits à l'image de l'Europe et propres à propager sa civilisation dans le monde $)^{24}$. Contrairement à Rameau, LeroyBeaulieu s'est attardé sur le XIXe siècle, ce qui lui a valu une bonne note de la part de Levasseur, qui opine: «C'est surtout dans les grandes expériences de notre siècle que l'économie politique peut puiser des enseignements utiles à l'avenir.» ${ }^{25}$

Or Rameau a trop insisté sur le XVIIe siècle. Levasseur lui adresse ce reproche tout en reconnaissant que, dans son mémoire, «les origines de la population coloniale dans les siècles passés ont été finement traitées» ${ }^{26}$. Et, comme un écho, cet autre commentaire: «Dans les parties qu'il a touchées, il a déployé un talent incontestable, mais il est très incomplet, et partant son jugement est étroit. $\gg{ }^{27}$ Certaines des positions de Rameau reflètent toutefois un large consensus et agréent tout à fait à Levasseur: avec LeroyBeaulieu, par exemple, Rameau a mis en lumière le fait capital que «les colonies anglaises ont eu seules l'avantage de recevoir un grand courant continu de population laborieuse et morale» ${ }^{28}$. Levasseur voit dans les mémoires soumis au concours la confirmation de certains principes reçus en économie politique, tels: «l'avenir d'une colonie dépend en grande partie des qualités morales de la population qui la fonde», ou encore: «s'il n'est pas possible au début de doter une colonie de droits politiques, c'est-à-dire du gouvernement représentatif, il importe de lui laisser la liberté communale la plus entière pour permettre le plein essor de l'initiative individuelle.» ${ }^{29}$ Quant à la valeur morale des colons, qui les aide à surmonter les épreuves des premiers établissements, Levasseur s'oppose à la tendance de Rameau à laisser croire qu'il n'y a que

23 Des notes de Rameau, conservées dans les archives Decencière Ferrandière, portent à croire qu'il avait pensé à un autre plan que celui finalement adopté: Amérique du Nord et du Sud (colonies espagnoles, françaises, anglaises, hollandaises); - colonies à esclaves (Antilles, îles d'Afrique, colonies portuguaises d'Afrique et du Brésil); - colonies commerciales (Indes, îles de la Sonde, Mozambique, Sénégal); - colonies contemporaines (Australie, Algérie, Cochinchine, comptoirs de la Chine). Ce programme, parce que plus complet, aurait peut-être mieux convenu au jury.

24 E. Levasseur, «Rapport...», 499.

25 Ibid., 483.

26 Ibid., 508 .

27 Ibid., 483.

28 Ibid., 494

29 Ibid., 509. 
«la foi religieuse qui puisse communiquer l'énergie nécessaire» ${ }^{30}$. Rameau a eu le tort de n'étudier que les cas favorables à sa position et d'ailleurs il n'a même pas prouvé que le sentiment religieux «ait été dominant parmi [les colons] du Canada» ${ }^{31}$. Aussi Rameau paraît-il «soutenir une thèse plutôt que de prononcer un jugement impartial après examen complet de toutes les pièces du procès» ${ }^{32}$. En outre, il s'est tu sur l'une des causes de faiblesse de la NouvelleFrance aux yeux de Levasseur, «celle qui résulta de l'influence prépondérante des ordres religieux, de la grande étendue des biens de mainmorte qui en fut la conséquence, des dissentiments du gouverneur avec les Jésuites» ${ }^{33}$. L'académicien ne partage pas non plus son enthousiasme pour le système seigneurial, le blâmant de ne pas «s'apercevoir que ces institutions féodales de la vieille Europe ne pouvaient être, même au XVIIe siècle, qu'un obstacle au défrichement sur une terre vierge». En revanche, il admet implicitement un des arguments avancés par Rameau à l'appui de la thèse de la supériorité du colon français: "La nation anglaise, écrit le savant, ne s'est donc acclimatée en Amérique qu'avec lenteur; c'est un point curieux de l'histoire des colonies que l'auteur met bien en lumière.» D'après Levasseur toujours, Rameau justifie mal sa préférence pour les «colonies par transformation» et se refuse à admettre les difficultés quasi insurmontables que pose «la transformation des sauvages indigènes en peuples civilisés» ${ }^{34}$. On a le choix entre la subordination à l'espagnole, ce qui entraîne exploitation et oppression; la ségrégation à la jésuite, qui assouplit sans civiliser; et le libre contact qui, infailliblement, livre sans défense le faible au fort. Le critique reconnaît cependant que la transformation «est de nature à séduire un esprit généreux parce qu'il prend pour devise «améliorer sans détruire» ${ }^{35}$. Une dernière observation du rapporteur mérite d'être signalée. (On se souvient que les mémoires étaient soumis anonymement). Remarquant dans le travail de celui que nous savons être Rameau, «certains archaïsmes [qui] rappellent les manuscrits du XVIIe siècle», Levasseur se demande: «L'auteur n'appartiendrait-il pas à une de ces familles de colons qui ont depuis longtemps quitté la France et qui en ont conservé le vieux langage?» ${ }^{36}$ Si le style traduit le tour d'esprit, on tient peut-être là une des explications du succès de Rameau au Canada français: on lui aurait trouvé un air de famille.

30 Archives de l'Académie des Sciences morales et politiques, "Procès-verbal», séance du 19 mars 1870.

31 E. Levasseur, «Rapport...», 474.

Ibid., 475.

Ibid., 477.

Ibid., 481.

Ibid., 482.

36 Ibid., 473. 
Il ressort du présent article que le mémoire de Rameau est important même s'il est resté inédit. Il représente un temps fort de la réflexion de Rameau et une étape significative dans son évolution intellectuelle. Il offre l'occasion rare de surprendre l'élaboration d'une pensée. À Rameau, il a permis de poser les fondements de prises de position subséquentes. En effet, on a vu que ce travail l'a obligé à un immense effort de synthèse fondé sur une ambitieuse approche comparative. Par la suite, l'historien reprendra les conclusions de son mémoire sans dérouler à nouveau, avec le même luxe de détails, ses argumentations. D'ailleurs, il n'est pas rigoureusement exact de dire que ce mémoire est resté inédit. Rameau publiera bon nombre d'articles et de communications, diffusés au Québec, dont l'inspiration - quand ce n'était pas des phrases entières - a manifestement été puisée dans le mémoire ${ }^{37}$. L'oeuvre appartient donc à l'histoire de notre vie intellectuelle en même temps qu'à celle des relations culturelles francoquébécoises. Elle interprète le passé afin de ménager des échappées sur l'avenir, de fonder des espoirs. Si plus tard les événements contredisent l'interprétation, cela n'entraînera pas nécessairement de remises en question, tant l'imaginal, pour parler comme Cazenave et Auguet ${ }^{38}$, a d'emprise sur les consciences, une fois celles-ci soumises à ses sortilèges. Aux jours d'amertume, même convaincu d'erreur, l'idéologue, quel qu'il soit, aura la tentation de s'écrier, comme Auguste Bodard: "C'était le bon temps alors, le temps des illusions», - demi-aveu aussitôt rattrapé: «...le temps des illusions qui auraient pu devenir des réalités, si nous avions été soutenus.» ${ }^{39}$

37 On trouvera en appendice une liste indicative de ces publications et conférences. Il faut savoir aussi que Rameau a caressé le projet de remettre son travail sur le métier, mais en le morcelant en plusieurs études complémentaires. En 1880, il travaillait à une étude comparée des procédés de colonisation de la France et de l'Angleterre en Amérique. Cet ouvrage devait être suivi par d'autres sur les Puritains du Massachusetts et les Patronats hollandais. Ces projets n'on pas abouti: la deuxième édition d'Une colonie féodale et d'autres intérêts les ont supplantés (Archives du Séminaire de Québec, fonds Casgrain, lettres, v. 9 , no 108, Rameau à H.-R. Casgrain, 24 avril 1880 ). On trouvera dans le fonds Rameau, au Centre d'études acadiennes, un plan sommaire et des notes en vue d'un autre ouvrage sur la «Propriété foncière en Angleterre et les colonies anglaises» (cote 2.2-13).

$38 \mathrm{M}$. Cazenave et R. Auguet, Les Empereurs fous, Essai de mythanalyse historique (Paris, Imago, 1981).

${ }_{39}$ ANQ (Montréal), fonds Proulx, A. Bodard à J.-B. Proulx, 15 nov. 1900. Français établi au Canada, agent d'immigration, Bodard a partagé les convictions des Rameau et des Reclus sur le destin des Canadiens français, la colonisation et l'immigration française au Canada. Il a été le fondé de pouvoir des actionnaires français dans l'Affaire du Témiscamingue. Il est assez représentatif des «amis du Canada». Voir P. Trépanier, Siméon Le Sage, Un haut fonctionnaire québécois face aux défis de son temps (1867-1909) (Montréal, Bellarmin, 1979), 145-54; Idem, "Siméon Le Sage et l'affaire du Témiscamingue (1884-1902)», Revue de l'Université d'Ottawa, 47 (1977): 365-76. 


\section{APPENDICE}

1 Conférence sur les Canadiens au Cercle catholique du Luxembourg en 1868. On n'en a ni le titre, ni le texte. Cf. Cercle catholique du Luxembourg, Séance solennelle d'inauguration des nouveaux salons du cercle..., 18 (1868-69) : 32 : «... à la suite de M. Rameau, vous avez recherché les traces ineffaçables que nos pères laissèrent au Canada».

2 Intervention sur le «Rapport de M. le comte de Moriana sur la Monographie du paysan de la province de Santander (Espagne). - Discussion sur l'émigration», Bulletin de la Société d'économie sociale (désormais Bull.), 3 (1869-71): 172-74.

3 «Rapport sur le rôle de la race française dans la colonisation du Canada», Bull., 4 (1872-75) : 45-63. Rameau évoque ici sommairement les thèses qu'il défend dans «Du système colonial des peuples modernes». On remarquera qu'il prenait soin d'assurer la diffusion de ses publications au Canada à travers son réseau de correspondants ou en en obtenant la reproduction dans nos journaux et revues. Voir sa lettre à Siméon Le Sage, ANQ (Québec), fonds S. Le Sage, 28 fév. 1873.

4 Intervention sur le «Rapport sur le rôle social de la grande propriété au Mexique et sur quelques déductions applicables à l'Algérie, par Édouard Pierron», Bull., 4 (1872-75) : 90-95.

5 Conférence au Cercle catholique du Luxembourg, 19 mars 1877. Le sujet était : «Les Français au Canada depuis la découverte jusqu'à nos jours». La conférence est résumée dans G.-A. Drolet, «Correspondance européenne». L'Opinion publique, 12 avril $1877: 169$.

6 Conférence au Cercle catholique du Luxembourg sur l'état primitif des colonies anglo-américaines, 17 janv. 1879. Rameau donne les grandes lignes de sa conférence dans une lettre à l'abbé Casgrain, Séminaire de Québec, fonds Casgrain, lettres, v. 9, no 86, 30 janv. 1879. Le Centre d'études acadiennes de l'Université de Moncton conserve, sous la cote 2.2-13, des notes ayant vraisemblablement servi à cette conférence.

7 Conférence au Cercle catholique du Luxembourg, 3 mai 1886. Titre : «De la colonisation de l'Amérique du Nord par la France et par l'Angleterre». Le texte de la conférence est perdu, mais un billet d'invitation, dans les archives de la famille Decencière Ferrandière, à Paris, précise le titre et la date.

8 «De la constitution de la propriété et de la distribution du sol dans l'Amérique du Nord, au moment de sa colonisation par les Hollandais, les Anglais et les Français"), Bulletin du Comité des travaux historiques, Section des sciences économiques et sociales (Paris), 1886 : 95. Le Bulletin ne fournit qu'un résumé, mais le brouillon, de 36 pages, se trouve au Centre d'études acadiennes, 2.2-13. Il se peut que d'autres pièces, apparaissant sous les cotes 2.4-6, 2.4-7 et 2.11-18, soient aussi des brouillons de cette communication.

9 Intervention au cours de la discussion de la communication d'Ernest Michel, au congrès annuel des Sociétés savantes de France, sur «L'expansion de la France, sa nécessité, les moyens de la développer», Bulletin du Comité des travaux historiques, Section des sciences économiques et sociales, $1892: 83$.

10 «Éléments qui ont concouru à former la population des États-Unis d'Amérique», Bulletin du Comité des travaux historiques, Section des sciences économiques et sociales, 1892 : 90. 
11 "L'expansion des Franco-Canadiens et la colonisation française en Algérie, Études comparées d'histoire contemporaine», La Réforme sociale, 2e série, 8 (1889) : 654-67. Communication signalée dans les journaux du Canada français, par exemple, dans l'Évangéline du 2 janvier 1890.

12 Conférence au Cercle des ouvriers, Paris. Date inconnue. La conférence porte sur les causes de la grandeur et de la décadence des colonies françaises. Voir Centre d'études acadiennes, fonds Rameau, 2.4-6. 